\title{
Report of the Fifth European Meeting on Psychosocial Aspects of Genetics
}

The Fifth European Meeting on Psychosocial Aspects of Genetics was held in Rome on 26-28 September 1996, attended by around 120 delegates from 10 different European countries, the USA, and Australia. It is a biannual event, with previous conferences having been held in Groningen (The Netherlands), Leuven (Belgium), Nottingham (UK), and Heidelberg (Germany). The aim of these meetings is to provide a forum for the discussion of psychological and social aspects of practice in the rapidly developing field of clinical genetics.

The opening address to the conference was given by Seymour Kessler (California), psychotherapist, renowned author on psychological issues in genetic counselling, and pioneer in the development of training programmes for non-medical genetic counsellors in the USA. The theme of the address was the long standing directiveness/non-directiveness debate, likened by Kessler to a "chronic illness that won't go away". He reminded the audience that nondirectiveness amounts to much more than the withholding of advice. In Kessler's view, attention must be given to procedures aimed at promoting client autonomy, with a requirement that genetic counselling be "opened up for public scrutiny". He proposed that an analysis of "what goes on" within the genetic counselling session could indicate a need to limit seriously the amount of information given, making room for the development of more sophisticated counselling skills to enable clients to make their own decisions. This was well received, preaching as he was to the converted!

Nearly 50 papers and 40 posters were presented during a fully occupied two and a half days. Findings of a diverse range of research studies and clinical programmes were presented, covering various themes of interest and relevance to those concerned with the psychological and social impact of genetic disorders and genetic testing.

\section{Predictive genetic testing for late onset disorders and inherited cancers}

Mansie (Melbourne) described a five year experience of a presymptomatic testing programme for those at risk for Huntington's disease. One unique feature, which has yet to be evaluated systematically, involved asking the counsellees and partners to keep a written "journal of feelings" for two weeks before the predictive test. During one week, they were asked to record their feelings while imagining they had a "normal" result, with the other week spent recording their feelings with an imagined "affected" result. A Belgian follow up study reported by Decruyenaere (Leuven), using preand post-test psychometric measurement, concluded that pre-test psychological health and not test results was the best predictor of post-test psychological function.

Early results of predictive testing for inherited cancer susceptibilities and syndromes, such as hereditary breast and ovarian cancer (HBOC) and multiple endocrine neoplasia type 2A (MEN 2A), suggest that those undergoing such testing are more emotionally vulnerable than those who have undergone HD presymptomatic testing and hence may experience more emotional problems. Grosfeld (Utrecht) described the increased vulnerability of women carriers of MEN 2A (and female partners of male carriers) to adverse psychological reactions over their male counterparts, while Frets (Rotterdam) found evidence of denial and detrimental rationalisation in women who were at risk of being HBOC gene carriers. Two-thirds of the 57 women in the latter Dutch study thought that their problems would not increase if they were found to be gene carriers, many using the apparent rationalisation that "a cure will be found".

Some papers described how patients' own assessments of personal cancer risk often fail to follow mendelian laws and are heavily influenced by other factors. This was clear in Green's study of 46 women attending a family cancer clinic in Cambridge. Beliefs about the non-genetic causes of cancer (smoking, stress, diet, personality) were prevalent. Only a few women had a mendelian concept of genetics. Green concluded that the explanation provided by the clinical geneticist may need to be "compatible" with a woman's beliefs in order for her to be able to take this "on board". The disparity between mendelian explanations and women's beliefs may explain the reluctance of many patients in receipt of "good news" to give up screening. Such a disparity may also throw light on why Ashkenazi Jewish women in a Jerusalem cancer clinic (described by Sagi) tended to overestimate their risk of breast cancer. JulianReynier (Marseille), in a study to investigate the effect of cancer genetic consultations on clients' perceptions of cancer risk and on their anxiety, also found that cancer risk perception post-consultation was determined by previous beliefs. 
Coping with the burden of genetic disease A Belgian study of the psychosocial impact of Marfan syndrome in 16-30 year old affected patients (Van Tongerloo and De Paepe) showed the significant social burden of being "different" and the domination of health concerns and potential pregnancy complications. Saviolo-Negrin (Padua) studied the parents of 20 haemophiliacs, finding the burden of anxiety and depression to be significantly more in mothers than in fathers, while Hall's (Manchester) study of mothers of dysmorphic children showed some of the benefits of a diagnosis. These included facilitating the acceptance of a child's disabilities.

\section{Carrier testing and genetic screening}

A study of the long term psychological effects of population based cystic fibrosis screening in the UK was reported by Marteau (London). The study sample comprised all the $435 \mathrm{CF}$ carriers identified in six UK centres and 870 matched "screen negative" testees. The main problem appeared to lie in the poor retention of information regarding test results. Half of the "screen negative" respondents believed that they definitely did not carry the CF gene, while $16 \%$ of carriers thought their carrier status was likely rather than proven.

The implications of screening for fragile $\mathrm{X}$ syndrome in The Netherlands was considered in a study by Tibben and colleagues (Rotterdam). A total of 1567 undiagnosed subjects in regional institutions and special schools were tested, of whom 11 were found to have the FMR1 gene. Most parents of young affected children reported that the diagnosis of fragile $\mathrm{X}$ had proved helpful. They had developed a greater understanding of the child's behaviour, with which they felt better able to cope, and subsequently held more realistic expectations of their child's potential. The Rotterdam study described a widespread acceptance of fragile X screening both among parents and staff of the relevant institutions.

\section{Evaluation of counselling and teaching a counselling approach}

Van Zuuren (Amsterdam) reminded us of the major degree of uncertainty in the information provided during genetic counselling. In their cohort of 30 counselling sessions, an inconclusive genetic diagnosis was "the rule rather than the exception". The best that could be offered to consultands in most sessions was risk estimation, usually in the absence of any prophylactic or therapeutic measure. As this is at odds with the need for (and expectations of) certainty which many clients bring to a genetics department, van Zuuren appealed for public education aimed at correcting false assumptions of what genetic counselling can offer.

The use of audiotape to help patient/parent recall in oncology and paediatrics has already been reported as beneficial. However, the use of this simple technology has been largely unexplored in clinical genetics, despite the emphasis placed on information giving within the specialty. A study by Curtis (Southampton) aimed to examine the use of audiotaped recordings on a client's information recall. Thirty-seven counselling sessions were recorded, and clients were randomly allocated to receive or not receive the tape to take home. Those who received the tape remembered more genetic information when this was assessed some time later. Although a small study, Curtis's findings suggest that the use of audiotape is acceptable to clinicians and clients alike, is a financially viable proposition, and may improve both information recall and the passage of genetic information within a family.

\section{Public understanding of and education about genetics}

Hasan's study of consanguinity among a section of the Manchester Pakistani population showed a low level of awareness of the genetic implications of intrafamilial marriage. Although the majority of those interviewed believed the figures for increased congenital malformation rates given by the researcher, many felt that the social advantages of first cousin marriage outweighed the risks.

The importance of educating both public and professional audiences about genetics was highlighted in a session chaired by Gerry Evers-Kiebooms, who showed one of a series of educational videos produced by the Centre for Human Genetics in Leuven. This showed how modern computer graphics can be used to enhance a clear and thoughtful visual presentation of genetic principles, and the potential for video to provide an introduction to some of the psychosocial and ethical issues in genetic practice.

\section{Reproductive decision making and prenatal diagnosis}

Zoeteweij described the Leiden experience of 60 prenatal tests for HD in 37 women. One of the objectives of this study was to investigate the decision making process and to identify psychological distress after termination of pregnancy. Preliminary results indicated that prospective parents with the HD gene minimised the significance of their own genetic status and denied their own childhood experiences with an affected parent. Their desire to have children was supported by their partners, who were determined to gratify the needs of the gene carriers. Prospective children were regarded by some gene carriers as a source of future support for the non-carrier parent.

The conference ended with a "Round Table" session, the aim of which was to describe and discuss the input of non-medical genetic professionals to the organisation and provision of genetic counselling services in each participating country. Lauren Kerzin-Storrar updated the conference on the progress of the MSc in Genetic Counselling course in Manchester and the main findings of the UK Association of Genetic Nurses and Counsellors "Scope of Practice" Working Party. Gioia Jacopini, the conference organiser, reported the findings of a study of genetic services in Italy, where $54 \%$ of genetic counsellors are medical and $46 \%$ nonmedical. The majority of Italian geneticists felt inadequately trained to deal with psychosocial 
problems. From The Netherlands, Hilda Van Spijker spoke on behalf of the seven clinical genetics centres and the 24 non-medical co-workers currently in post. In Australia, genetic counsellors have existed for nine years, mostly as graduates of the diploma or Masters degree courses, who have completed the required two years of full time supervised experience.

The participants enjoyed the well organised and multifaceted meeting in the historical centre of Rome. Nevertheless, some regretted the strong emphasis on the presentation of research findings and the fact that the comprehensiveness of the programme did not allow time for participatory workshops. The need for thematic workshops, particularly felt by the genetic co-workers from a background of social work and nursing, was well recognised at the meeting and is being addressed by the Scientific Committee for the 1998 conference, to be held in Paris.

CHRIS BARNES

Genetic Counsellor, South Thames Regional Genetics Centre (East), Guy's Hospital, London SE1 9RT, UK

THERESA MARTEAU

Professor of Health Psychology, Psychology E Genetics Research Group, UMDS, Guy's Campus, Guy's Hospital, London SE1 9RT, UK

GERRY EVERS-KIEBOOMS

Professor of Psychology, Psychosocial Genetics Unit, Centre for Human Genetics, University of Leuven, Herestraat 49, 3000 Leuven, Belgium 